\title{
The Australia Telescope Large Area Survey: 2.3 GHz observations of ELAIS-S1 and CDF-S
} \section{Spectral index properties of the faint radio sky $\star$}

\author{
P.-C. Zinn ${ }^{1,2}$, E. Middelberg ${ }^{1}$, R. P. Norris ${ }^{2}$, C. A. $\operatorname{Hales}^{3,2}$, M. Y. Mao ${ }^{2,4,5}$, and K. E. Randall ${ }^{2,3}$ \\ 1 Astronomical Institute of Ruhr-University Bochum, Universitätsstraße 150, 44801 Bochum, Germany \\ e-mail: zinn@astro.rub.de \\ 2 CSIRO Astronomy \& Space Science, PO Box 76, Epping, NSW 1710, Australia \\ 3 Sydney Institute for Astronomy, School of Physics, The University of Sydney, NSW 2006, Australia \\ 4 Australian Astronomical Observatory, PO Box 296, Epping, NSW 1710, Australia \\ 5 School of Mathematics \& Physics, University of Tasmania, Private Bag 37, 7001 Hobart, Australia
}

Received 5 April 2012 / Accepted 22 June 2012

\section{ABSTRACT}

\begin{abstract}
Context. The Australia Telescope Large Area Survey (ATLAS) aims to image a $7 \mathrm{deg}^{2}$ region centred on the European Large Area ISO Survey - South 1 (ELAIS-S1) field and the Chandra Deep Field South (CDF-S) at $1.4 \mathrm{GHz}$ with high sensitivity (up to $\sigma \sim 10 \mu \mathrm{Jy}$ ) to study the evolution of star-forming galaxies (SFGs) and Active Galactic Nuclei (AGN) over a wide range of cosmic time.

Aims. We present here ancillary radio observations at a frequency of $2.3 \mathrm{GHz}$ obtained with the Australia Telescope Compact Array (ATCA). The main goal of this is to study the radio spectra of an unprecedented large sample of sources ( 2000 observed, $\sim 600$ detected in both frequencies).

Methods. With this paper, we provide $2.3 \mathrm{GHz}$ source catalogues for both ATLAS fields, with a detection limit of $300 \mu \mathrm{Jy}$ (equivalent to $4.5 \sigma$ in the ELAIS-S1 field and $4.0 \sigma$ in the CDF-S). We compute spectral indices between $1.4 \mathrm{GHz}$ and $2.3 \mathrm{GHz}$ using matchedresolution images and investigate various properties of our source sample in dependence of their spectral indices.

Results. We find the entire source sample to have a median spectral index $\alpha_{\text {med }}=-0.74$, in good agreement with both the canonical value of -0.7 for optically thin synchrotron radiation and other spectral index studies conducted by various groups. Regarding the radio spectral index as indicator for source type, we find only marginal correlations so that flat or inverted spectrum sources are usually powered by AGN and hence conclude that at least for the faint population the spectral index is not a strong discriminator. We investigate the $z-\alpha$ relation for our source sample and find no such correlation between spectral index and redshift at all. We do find a significant correlation between redshift and radio to near-infrared flux ratio, making this a much stronger tracer of high- $z$, radio sources. We also find no evidence for a dependence of the radio-IR correlation on spectral index.
\end{abstract}

Key words. galaxies: active - catalogs - galaxies: evolution - radio continuum: galaxies - surveys

\section{Introduction}

Large-scale radio surveys are the primary instrument in the toolbox of contemporary astronomy to investigate demography and evolution of active galaxies across cosmic time. Consequently, there have been numerous attempts to use this tool, whether in a wide, shallow layout (e.g. the National Radio Astronomy Observatory Very Large Array Sky Survey, Condon et al. 1998) or in a deep, but more pencil beam-like layout such as for the Cosmic Evolution Survey (COSMOS, Schinnerer et al. 2010) or even the Lockman Hole survey by Owen \& Morrison (2008), the most sensitive radio observation to date.

The Australia Telescope Large Area Survey (ATLAS, Norris et al. 2006; Middelberg et al. 2008) attempts to break this dichotomy by obtaining deep images $(\sigma \sim 10 \mu \mathrm{Jy}$ in the final data release 3) over an unprecedented large area of sky. Distributed over the well-studied Chandra Deep Field South (CDF-S) and the European Large Area ISO Survey - South 1 (ELAIS-S1) field, the total area covered by ATLAS is approximately seven

* Full Table 2 is only available at the CDS via anonymous ftp to cdsarc.u-strasbg.fr (130.79.128.5) or via

http://cdsarc.u-strasbg.fr/viz-bin/qcat?]/A+A/544/A38 square degrees. This allows us to study the faint radio sky without being affected by cosmic variance (Moster et al. 2011). Furthermore, the wealth of ancillary photometric data ranging across the entire electromagnetic spectrum (e.g. from SWIRE, COMBO-17 or GEMS Lonsdale et al. 2003; Wolf et al. 2003; Rix et al. 2004) as well as numerous spectroscopic campaigns (Sacchi et al. 2009; Popesso et al. 2009; Balestra et al. 2010; Mao et al. 2012) enables one to conduct detailed studies of radiodetected objects.

In this paper, we focus on radio spectral properties of the ATLAS source sample. The main ATLAS data releases are DR 1 by Norris et al. (2006) and Middelberg et al. (2008), DR 2 by Hales et al. (in prep.), and DR 3 by Banfield et al. (in prep.). These data consist of $1.4 \mathrm{GHz}$ observations as for most contemporary radio surveys, and we here supplement these data with observations at $2.3 \mathrm{GHz}$. The main goal of this is to calculate spectral indices $\alpha \equiv$ $\log \left(S_{1.4 \mathrm{GHz}} / S_{2.3 \mathrm{GHz}}\right) / \log (1.4 \mathrm{GHz} / 2.3 \mathrm{GHz})$ for all sources detected at both wavelength and so to characterise their radio emission mechanism, source type and dependence on other spectral quantities. Even though many blind surveys of extragalactic radio sources are available already, only few studies have been 
carried out which characterise the radio spectra of a large source population by means of their spectral index. For example, Bondi et al. (2007) and Ibar et al. (2009) use supplementary $610 \mathrm{MHz}$ data to calculate spectral indices at frequencies below $1.4 \mathrm{GHz}$ with the main aim to identify steep spectrum sources which are regarded as good candidates for being high-redshift objects (see Sect. 4.3). The most comprehensive study of spectral indices between $1.4 \mathrm{GHz}$ and a higher frequency was conducted by Prandoni et al. (2006) using $5 \mathrm{GHz}$ Very Large Array (VLA) data in the Australia Telescope ESO Slice Project survey field. With a sensitivity of $\sim 70 \mu \mathrm{Jy}$ over an area of one square degree, their source sample consists of approximately 100 objects which are detected at both frequencies, so our sample exceeds this by a factor of six.

According to Komatsu et al. (2011), we adopt a flat $\Lambda$ CDM cosmology with $H_{0}=70.2 \mathrm{~km} \mathrm{~s}^{-1} \mathrm{Mpc}^{-1}$ and $\Omega_{\Lambda}=0.725$ throughout this paper.

\section{Observations and imaging}

In the following description, we give the actual numbers for all things concerning the ELAIS-S1 field first, followed by the numbers for the CDF-S in parentheses. If there is only a single number given, it is approximately equal for both fields. Observations at $2.3 \mathrm{GHz}$ for the ATLAS fields were carried out between December 2006 and March 2007 on 52 separate days where the ATCA was only in its 750B and 750C configurations. This resulted in a substantially lower resolution than at $1.4 \mathrm{GHz}$ since the longest baselines used for imaging at $2.3 \mathrm{GHz}$ was $750 \mathrm{~m}$, or $5.7 \mathrm{k} \lambda$, resulting in an angular resolution of $33^{\prime \prime}\left(57^{\prime \prime}\right)$, compared to $30 \mathrm{k} \lambda$ at $1.4 \mathrm{GHz}$. The ATCA primary beam at a frequency of $2.3 \mathrm{GHz}$ has a $F W H M$ of 21 arcmin, therefore 76 (88) overlapping pointings were observed in a hexagonal lattice with an average integration time of $6.17 \mathrm{~h}(4.13 \mathrm{~h})$ per pointing (compared to approx. $12 \mathrm{~h}$ per pointing for the $1.4 \mathrm{GHz}$ image) to cover the entire area of the $1.4 \mathrm{GHz}$ maps.

For phase calibration, which was done approximately every 45 min, PKS 0022-423 (PKS 0237-233) was observed as secondary calibrator. Flux calibration was done for both fields using PKS 1943-638 which was observed at least once a day for $10 \mathrm{~min}$. As for $1.4 \mathrm{GHz}$, the ATCA provides two independent frequency bands with a bandwidth of $128 \mathrm{MHz}$ divided into 33 channels. During the first two days of observations for the ELAIS-S1 field, the two bands were centred at $2.368 \mathrm{GHz}$ and $2.560 \mathrm{GHz}$ in an attempt to reduce local Radio Frequency Interference (RFI). The fluxes density of the primary calibrator, PKS 1934-638, was assumed to be $11.589 \mathrm{Jy}$ and $10.932 \mathrm{Jy}$, respectively. Because using a higher frequency for the upper band did not significantly decrease the RFI, the remaining time was observed with the standard band configuration, meaning that the upper band was lowered in central frequency to $2.496 \mathrm{GHz}$. There, the primary calibrator was assigned to have a flux density of $11.138 \mathrm{Jy}$. We note that the final images were made with the entire data set.

The calibration of the data were carried out using standard Miriad (Sault et al. 1995) tasks. ATLOD was used to convert the raw data from RPFITS format to the native Miriad format. For the further calibration, both channels at the end of the bands were discarded due to a significant lack of sensitivity, hence a data set containing two times 13 channels with $8 \mathrm{MHz}$ bandwidth each was obtained, yielding a net bandwidth of $208 \mathrm{MHz}$. Fortunately, our data do not suffer from self-interference typical for the ATCA. These so-called "birdies" occur within the samplers at integer multiples of $128 \mathrm{MHz}$. The 19th harmonic at $2.432 \mathrm{GHz}$ fortunately lies exactly between our two bands and therefore does not affect our data. After bandpass-calibration, RFI was removed using Pieflag (Middelberg 2006), a tool that compares statistics from channels with no or little RFI contamination to the other channels in the data and looks for outliers and sections with high noise. Bandpass calibration was carried out using the observations of PKS 1934-638. Phase calibration was done using the regular scans of the secondary calibrators, and then amplitude calibration was carried out using PKS 1934-638. The data were split into the individual pointings which were imaged separately using uniform weighting. This provides a higher resolution at the cost of lower sensitivity than natural weighting. The cell size was set to $6^{\prime \prime}\left(3^{\prime \prime}\right)$. Because of the low fractional bandwidth $(<10 \%)$, a normal CLEAN procedure was used (in contrast to the Miriad multi-frequency clean implementation MFCLEAN for the $1.4 \mathrm{GHz}$ data) for deconvolution. Also the dynamic range in the pointings was sufficiently low as to not require any special deconvolution procedures. Cleaning was carried out using 2000 iterations, after which only marginal sidelobes around the two strongest sources remained, and the effects of clean bias should be negligible. After cleaning, the models were convolved with a restoring beam of $33.56^{\prime \prime} \times 19.90^{\prime \prime}$ $\left(57.15^{\prime \prime} \times 22.68^{\prime \prime}\right)$ at a position angle of $-1.32 \mathrm{deg}(-1.90 \mathrm{deg})$. The final mosaics were produced using Miriad's LINMOS task to account for primary beam attenuation and overlapping regions of the individual pointings. As a result, the edges of the final images exhibit a significantly higher noise level than the central regions (see Fig. 1).

\section{The source catalogue}

Source extraction from the final $2.3 \mathrm{GHz}$ images was performed in two steps: first, all known $1.4 \mathrm{GHz}$ sources from ATLAS DR 2 (Hales et al., in prep.) were used as an input catalogue to search for $2.3 \mathrm{GHz}$ counterparts. This catalogue contains a total of 2240 individual $1.4 \mathrm{GHz}$ sources across both the ELAIS-S1 and the CDF-S of which 631 could be reliably detected at $2.3 \mathrm{GHz}$. To identify these counterparts, the $2.3 \mathrm{GHz}$ image was inspected by eye at the location of every individual $1.4 \mathrm{GHz}$ source. This was done by half-automatically browsing through an input catalogue, simultaneously displaying the corresponding regions in the $1.4 \mathrm{GHz}$ and $2.3 \mathrm{GHz}$ images and, if a $2.3 \mathrm{GHz}$ counterpart was found, fitting a Gaussian to it and recording all fit parameters in an output catalogue. Thus, no blind search was conducted at $2.3 \mathrm{GHz}$, allowing the inclusion of sources fainter than the typical $5 \sigma$ cutoff in the catalogue. The lowest signal-to-noise ratio among the $2.3 \mathrm{GHz}$ sources is 3.9.

In a second step, we checked for " $2.3 \mathrm{GHz}$-only" sources. Those sources are unlikely to occur because of the noise levels of the $1.4 \mathrm{GHz}$ and $2.3 \mathrm{GHz}$ images. Since the faintest $1.4 \mathrm{GHz}$ sources in the DR 2 catalogue are as faint as $100 \mu \mathrm{Jy}$, whereas the faintest sources in $2.3 \mathrm{GHz}$ are three times brighter, a $2.3 \mathrm{GHz}$ only source would have a highly inverted spectral index of $\alpha \geq 2.4$. Also, we note that, due to the temporal offsets between the $1.4 \mathrm{GHz}$ and $2.3 \mathrm{GHz}$ observations, possible variability of some radio sources may lead to flawed spectral indices. Nevertheless, we used MIRIAD's SAD task to automatically produce a $2.3 \mathrm{GHz}$ catalogue with a detection threshold of $5 \sigma$ and matched the sources obtained that way to the $2.3 \mathrm{GHz}$ catalogue obtained by cross-matching to $1.4 \mathrm{GHz}$ sources. All sources identified by SAD could be matched to sources previously found as $1.4 \mathrm{GHz}$ counterparts, hence not a single $2.3 \mathrm{GHz}$-only source was found. 

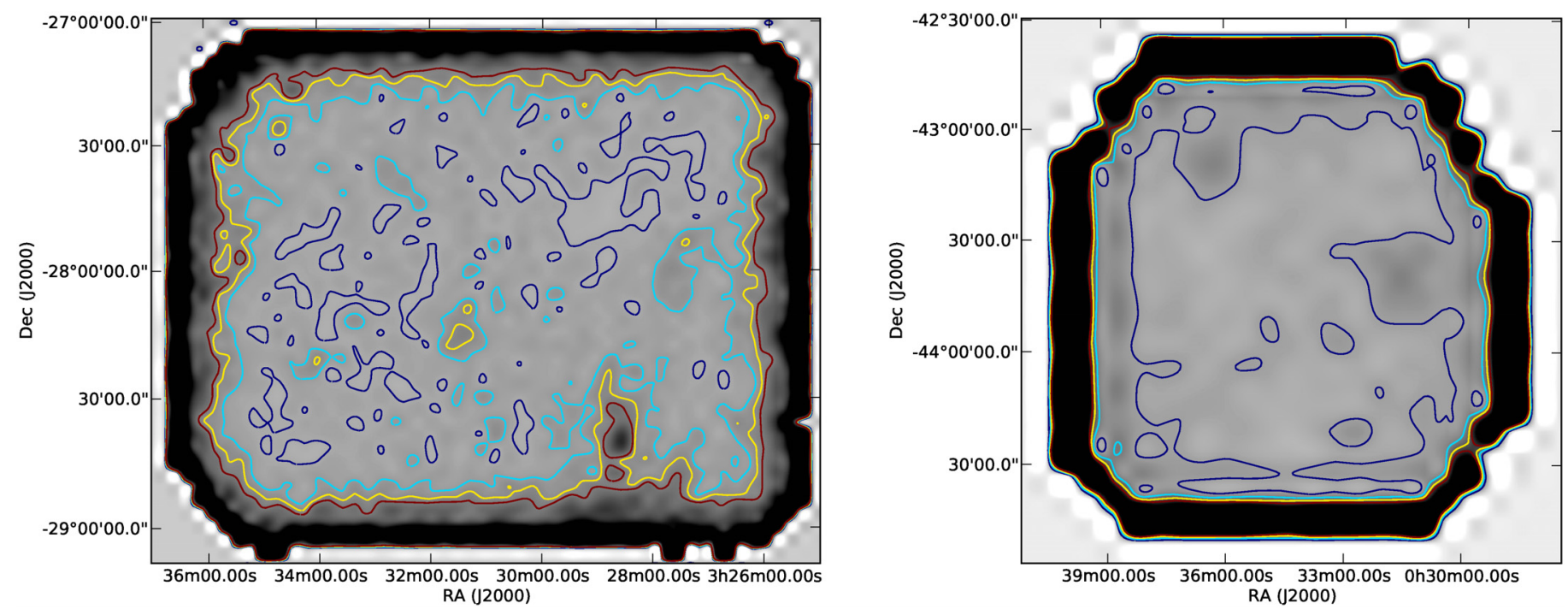

Fig. 1. 2.3 GHz noise maps of both ATLAS fields: CDF-S (left) and ELAIS-S1 (right). Contours start at $70 \mu \mathrm{Jy}_{\text {beam }}{ }^{-1}$ (dark blue contour line) and increase by a constant value of $40 \mu \mathrm{Jy}_{\text {beam }}{ }^{-1}$.

Table 1. Parameters of the observations.

\begin{tabular}{lccccc}
\hline \hline Field & $N_{\text {pnt }}$ & $\begin{array}{c}t_{\text {int }} \\
\mathrm{h}\end{array}$ & $\begin{array}{c}\text { Area } \\
\mathrm{deg}^{2}\end{array}$ & $\begin{array}{c}\text { rms noise } \\
\mu \mathrm{Jy} / \text { beam }\end{array}$ & $\begin{array}{c}\text { Resolution } \\
\text { arcsec }\end{array}$ \\
\hline ELAIS-S1 & 76 & 6.17 & 2.77 & 70 & $33.56 \times 19.90$ \\
CDF-S & 88 & 4.13 & 3.63 & 80 & $57.15 \times 22.68$ \\
\hline
\end{tabular}

Notes. Given are the number of pointings per field, the average integration time per pointing, the total area covered by the pointings, the rms noise and the resolution of the final images. For comparison, we note that the corresponding typical rms noise levels of the $1.4 \mathrm{GHz}$ images are $31 \mu \mathrm{Jy}$ and for $38 \mu \mathrm{Jy}$ the ELAIS-S1 and CDF-S, respectively.

To compute spectral indices, the flux densities in $1.4 \mathrm{GHz}$ had to be re-measured because the resolutions of the $1.4 \mathrm{GHz}$ and $2.3 \mathrm{GHz}$ images differ significantly. Whilst the $1.4 \mathrm{GHz}$ observations included long baseline configurations of the ATCA (e.g. the $6 \mathrm{~A}$ and $6 \mathrm{~B}$ configurations), the $2.3 \mathrm{GHz}$ observations were obtained with the ATCA in $750 \mathrm{~B}$ and $750 \mathrm{C}$ configurations only. This leads to a resolution three to five times lower at $2.3 \mathrm{GHz}$ compared to the $\sim 10^{\prime \prime}$ resolution of the $1.4 \mathrm{GHz}$ observations, despite the shorter wavelength. Therefore, in order to avoid resolution effects, we convolved the original $1.4 \mathrm{GHz}$ images with a Gaussian kernel to match the resolution of the $2.3 \mathrm{GHz}$ images as given in Table 1. We then used the ATLAS DR 2 catalogue again as input to re-measure the $1.4 \mathrm{GHz}$ flux densities in the low-resolution images. Because the image sensitivity was reduced by a factor of $\sim 2$ by this convolution (which is equal to tapering the data in the $u v$-plane, so in fact weighting down long baselines), all sources in the low-resolution image should be a true subset of the hi-res sources and, therefore, all low-resolution sources should be detected when using the high-resolution catalogue as input sample.

During the re-measurement of the $1.4 \mathrm{GHz}$ flux densities using convolved images, a problem surfaced with the ELAIS$\mathrm{S} 1$ image. Unlike with the CDF-S convolved $1.4 \mathrm{GHz}$ image, where the procedure outlined above went well, the MIRIAD task imfit overestimated the flux densities by a significant amount (up to $50 \%$ ). This became obvious by inspecting residual images for sources with various flux densities and degrees of compactness. While the residuals looked good in the CDF-S, they showed bowls of negative flux in the ELAIS-S1 image. The cause of the effect remains unclear. The fitting routines in the software package CASA (Jaeger 2008) yielded similarly overestimated flux densities, so it appears that the effect is not caused by the fitting software, but is inherent in the images, and we had to work around this issue. This was done by iteratively fitting Gaussians to every individual source. The first Gaussian fit (which was always overestimating the flux density) was used as prior. The resulting residual image was then examined in terms of total flux and rms noise. If the total flux in the residual image (trimmed to a size twice as large as the restoring beam) was negative and the rms noise was deviating from the median rms in the region of the source by more than $10 \%$, the fit was discarded and replaced by a fit with a peak flux density $2 \%$ lower than in the previous fit. The residual image was again examined in a similar way. This procedure was iteratively repeated until the criteria (non-negative total flux and rms noise consistent with the rms in this region) were entirely met. As a last step, all final residual images were inspected by eye to assure the quality of the fit.

A further problem in calculating spectral indices for every $1.4 \mathrm{GHz}$ was introduced by the very different resolutions of the $1.4 \mathrm{GHz}$ and $2.3 \mathrm{GHz}$ observations. Because the resolutions differ by a large factor, as explained above, numerous sources which are clearly separated in the high-res images are blended together in the low-res images. Therefore, we chose to completely exclude such blended sources from our final catalogue if they could not be separated by simultaneously fitting two or more Gaussians. We note that this may bias our sample towards fainter sources since classic radio doubles (which are mainly excluded by the blending) are generally brighter than single, isolated sources.

The final catalogue was then constructed by merging the individual catalogues from ELAIS-S1 and CDF-S. This resulted in a final catalogue comprising 631 radio sources with clear detections and well-measured flux densities in both $1.4 \mathrm{GHz}$ and $2.3 \mathrm{GHz}$. A portion of the catalogue is shown in Table 2 for illustration.

We give an identifier for each source, the position, the original $1.4 \mathrm{GHz}$ flux density as measured by Hales et al. (in prep.), the re-measured $1.4 \mathrm{GHz}$ flux density using the convolved image, the $2.3 \mathrm{GHz}$ flux density as measured during the 


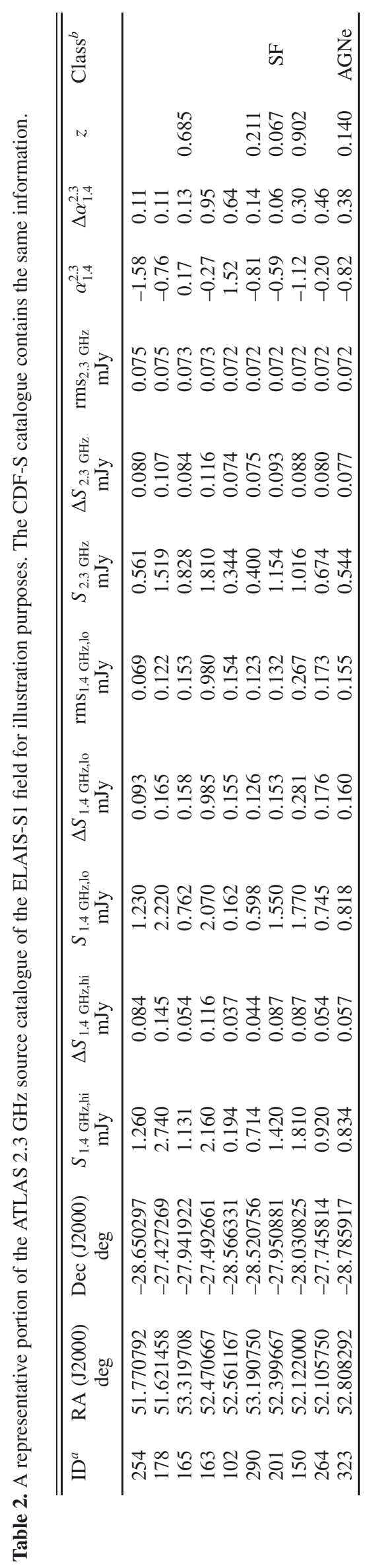

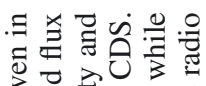

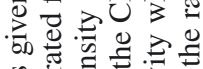

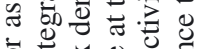

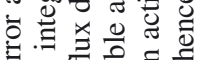

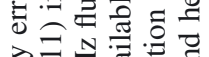

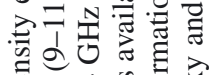

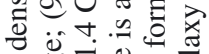$$
\text { 要 }
$$

cross-matching process and finally the spectral index calculated from the matched-resolution $1.4 \mathrm{GHz}$ and $2.3 \mathrm{GHz}$ flux densities. All flux densities are integrated quantities.

Errors for the flux densities were either taken from Hales et al. (in prep.) in case of the original $1.4 \mathrm{GHz}$ flux densities or calculated following Hopkins et al. (2003) for the low-resolution 1.4 GHz flux densities and the $2.3 \mathrm{GHz}$ flux densities, taking into account both the local rms noise at the source position as well as the quality of the Gaussian fit:

$\frac{\Delta S}{S}=\sqrt{\frac{\mu_{\mathrm{image}}^{2}}{S^{2}}+\frac{\mu_{\mathrm{fit}}^{2}}{S^{2}}}$

where $\mu_{\text {image }}$ quantifies the uncertainty in flux density due to the image noise and $\mu_{\text {fit }}$ represents uncertainties due to the fitting of a Gaussian. These two quantities can be calculated following Windhorst et al. (1984),

$\frac{\mu_{\text {image }}}{S}=\sqrt{\frac{\sigma^{2}}{S_{\text {peak }}^{2}}+C_{\mathrm{f}}^{2}+C_{\mathrm{p}}^{2}}$,

where $\sigma$ is the local rms noise, $C_{\mathrm{f}}$ the error in absolute flux calibration and $C_{\mathrm{p}}$ the error in flux due to eventual pointing errors. We here adopt a conservative value of $C_{\mathrm{f}}^{2}+C_{\mathrm{p}}^{2}=0.05^{2}$. The fitting error is calculated following Condon (1997),

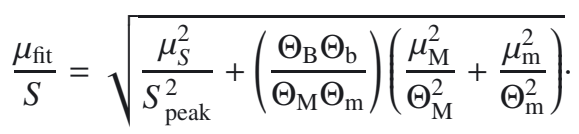

Here, $\Theta_{\mathrm{B}} \Theta_{\mathrm{b}}$ are the restoring beam's major and minor axes, whereas $\Theta_{M} \Theta_{m}$ represent the major and minor axes of the fitted Gaussian. The errors $\mu_{S}, \mu_{\mathrm{M}}$ and $\mu_{\mathrm{m}}$ stand for the errors of the fitted Gaussian's peak flux, major and minor axes. The error of the spectral index was finally calculated using simple Gaussian error propagation,

$\Delta \alpha=2 \sqrt{\left(\frac{\Delta S_{1.4 \mathrm{GHz}}}{S_{1.4 \mathrm{GHz}}}\right)^{2}+\left(\frac{\Delta S_{2.3 \mathrm{GHz}}}{S_{2.3 \mathrm{GHz}}}\right)^{2}}$,

where the factor of $2 \approx\left|[\log (1.4 \mathrm{GHz} / 2.3 \mathrm{GHz}) \cdot \ln (10)]^{-1}\right|$ arises from deriving the spectral index as defined in Sect. 1 with respect to the flux densities, which are the only quantities with uncertainties in this equation (the error in frequency is neglected).

Histograms illustrating the distribution of flux densities in the final catalogues are shown in Fig. 2, the relative flux density errors as a function of flux density are presented in Fig. 3. Further spectral index properties are investigated in the next section.

\section{Spectral index properties}

The distribution of spectral indices for our sample of 631 $1.4 \mathrm{GHz}$ sources with unambiguously identified $2.3 \mathrm{GHz}$ counterpart is shown in Fig. 4.

With a median spectral index of -0.74 our sample matches the canonical value for synchrotron radiation of -0.7 very well. However, we note that the distribution is broad with a median absolute deviation of 0.35 . We here use the median absolute deviation defined as MAD $\equiv \operatorname{median}_{\mathrm{i}}\left(\left|x_{\mathrm{i}}-\operatorname{median}_{\mathrm{j}}\left(x_{\mathrm{j}}\right)\right|\right)$ as a measure for the spread of a distribution since it is more robust against outliers than, for instance, the normal standard deviation, $\sigma$. In case of a perfect Gaussian distribution, $\sigma$ and MAD are related 

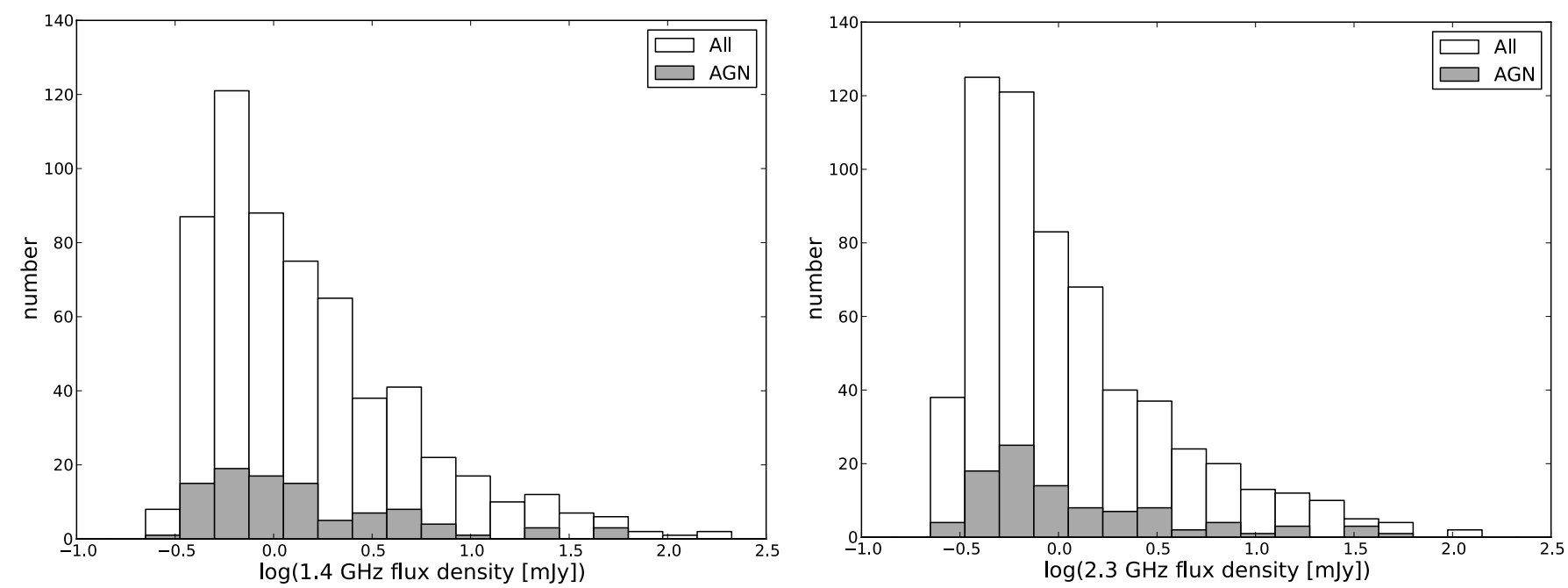

Fig. 2. Distribution of $1.4 \mathrm{GHz}$ (hi-res) and $2.3 \mathrm{GHz}$ flux densities in the final catalogue. AGN classifications of the sources are from Mao et al. (2012).

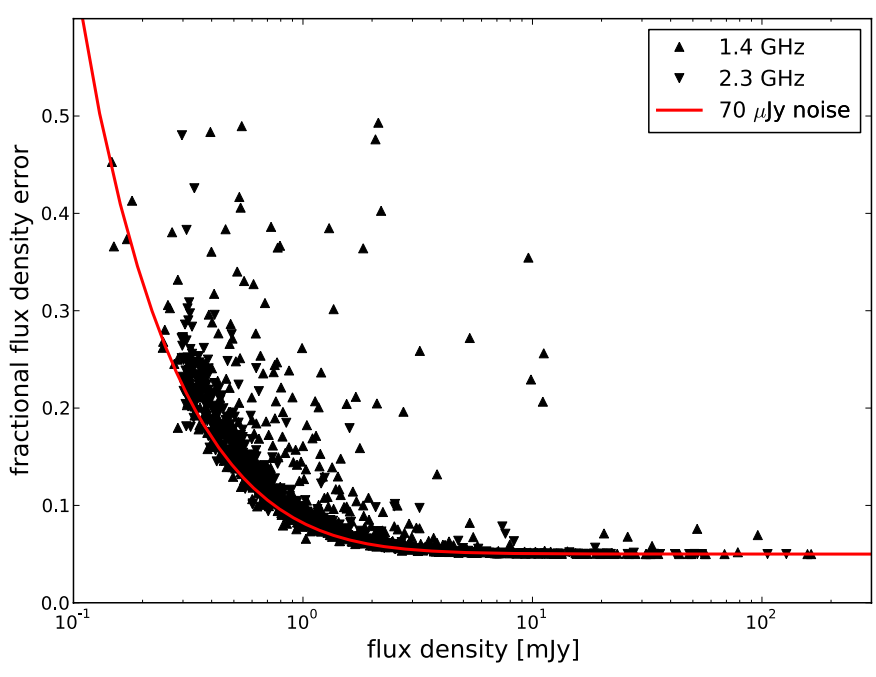

Fig. 3. The fractional flux density errors for all ELAIS-S1 and CDF-S sources as function of their flux density. The solid red line indictates how the fractional error should decrease with increasing flux density in an image with an overall rms noise of $70 \mu \mathrm{Jy}$ according to Eq. (2). A calibration error of $5 \%$ is assumed.

via $\sigma \approx 1.4826 \mathrm{MAD}$. This also compares nicely to other studies such as Prandoni et al. (2006) whose sample has MAD $=0.33$. The spread of the Ibar et al. (2009) sample is slightly smaller with MAD $=0.27$. This difference is due to the larger number of sources with flat or inverted spectra in our sample. We repeated the analysis including upper limits (following the survival analysis techniques developed in Feigelson \& Nelson 1985) for all $1.4 \mathrm{GHz}$ sources which do not have a $2.3 \mathrm{GHz}$ counterpart and are not affected by resolution effects (see Sect. 3). These sources do have a $2.3 \mathrm{GHz}$ flux density less than three times the rms noise at the respective position. The median spectral index then becomes slightly steeper with -0.81 and a MAD of 0.38 . The main reason for only having this slight change is the large difference between the $1.4 \mathrm{GHz}$ sensitivity (about $30 \mu \mathrm{Jy}$ ) and the $2.3 \mathrm{GHz}$ sensitivity $(70-80 \mu \mathrm{Jy})$. The number of sources detected at both $1.4 \mathrm{GHz}$ and $2.3 \mathrm{GHz}$ is 631 whereas there are 552 sources with an unambiguous upper limit at $2.3 \mathrm{GHz}$. We note that the errors of our spectral indices become quite large

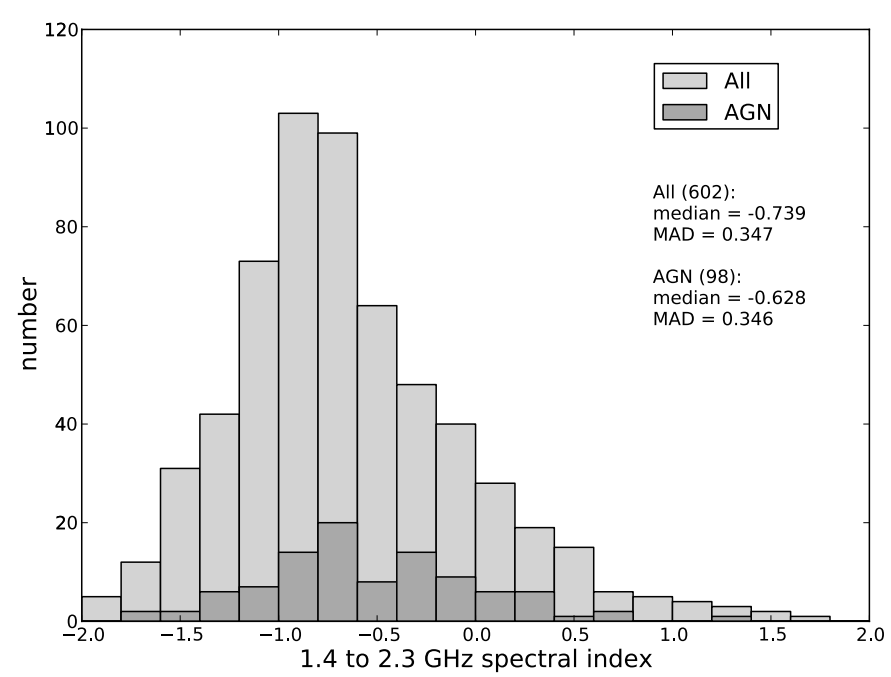

Fig. 4. Distribution of $1.4 \mathrm{GHz}$ to $2.3 \mathrm{GHz}$ spectral indices in the final catalogue for all sources with unambiguously detected $2.3 \mathrm{GHz}$ counterpart and both the $1.4 \mathrm{GHz}$ and $2.3 \mathrm{GHz}$ flux densities exceeding $0.3 \mathrm{mJy}$. AGN classifications of the sources are from Mao et al. (2012).

for low flux densities. This is because currently available radio data and the flux densities extracted from them have typical uncertainties at the $10 \%$ level. This is mostly due to either inhomogeneous data processing and calibration strategies as well as the widely adopted method of fitting 2-dimensional Gaussians to extract flux densities (see e.g. Condon 1997). Future largescale continuum surveys, such as ASKAP/EMU, are attempting to reach flux density errors of only $1 \%$ by developing both novel, fully automated calibration as well as source extraction techniques (Norris et al. 2011b).

\subsection{Spectral index vs. flux density}

A plot of the spectral index of our sources against their flux density is shown in Fig. 5. We point out that the sensitivities of our $1.4 \mathrm{GHz}$ and $2.3 \mathrm{GHz}$ data differ by a factor of about 3 . Therefore, progressively negative spectral indices cannot be measured anymore towards fainter $1.4 \mathrm{GHz}$ fluxes (as indicated 


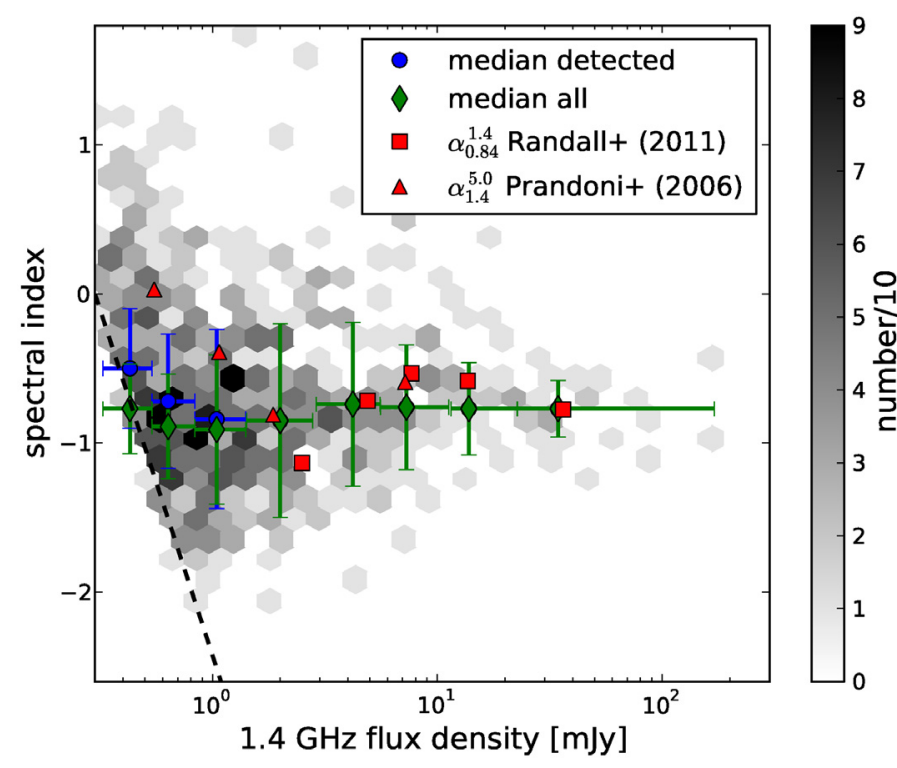

Fig. 5. Spectral index vs. flux density. The grey $2 \mathrm{D}$ histogram shows our sample of 631 sources with $1.4 \mathrm{GHz}$ and $2.3 \mathrm{GHz}$ detections. Since the sensitivity of the $2.3 \mathrm{GHz}$ observations is $\sim 3$ times lower than that the $1.4 \mathrm{GHz}$ data, the black dashed line indicates the minimum spectral index that could be measured with our data. Therefore, the median spectral indices (blue circles, $x$-error bars indicate the flux density bin size, $y$-error bars correspond to the MAD) for just the $2.3 \mathrm{GHz}$ detected sample show significantly flatter values towards lower flux densities. When including also upper limits from the $2.3 \mathrm{GHz}$ non-detected sources, this trend completely vanishes (blue diamonds). Hence we conclude that there is no evidence for a flattening of the mean spectral index with lower flux density.

by the black dashed line in Fig. 5). For instance, a source with $S_{1.4 \mathrm{GHz}}=200 \mu \mathrm{Jy}$ can only have a $2.3 \mathrm{GHz}$ counterpart when exhibiting a spectral index $\gtrsim 0.5$ since the $3 \sigma$ detection level of our $2.3 \mathrm{GHz}$ data is about $250 \mu \mathrm{Jy}$ (see Sect. 2). To account for this fact, we also included upper limits on spectral index for all $1.4 \mathrm{GHz}$ sources that unambiguously show no $2.3 \mathrm{GHz}$ counterpart (the upper limits were calculated using $S_{2.3 \mathrm{GHz}}<3 \sigma$ where $\sigma$ is the local rms noise for a point source) and are not affected by resolution issues (see Sect. 3, e.g. a close double in $1.4 \mathrm{GHz}$ that would be inseparable at the $2.3 \mathrm{GHz}$ resolution). With these upper limits, we calculated median spectral indices for different flux density bins to investigate a potential flattening of the mean spectral index towards fainter flux densities. As expected, the median spectral indices for only the detected sample (so the 631 sources with $2.3 \mathrm{GHz}$ counterparts) show a clear trend of flatter spectral indices with lower flux density (Fig. 5, blue circles). Including the upper limits into the median calculation, this trend vanishes immediately (Fig. 5, blue diamonds): the lowest flux density bin (about 300-600 $\mu \mathrm{Jy}$ ) is completely dominated by the upper limits and can hence not be included in further analysis whereas the two flux density bins at $700 \mu \mathrm{Jy}$ and $1 \mathrm{mJy}$ are now a even a little steeper than the ones at higher flux densities. Hence, we find no evidence for a flattening of the spectral index towards the lowest flux densities. This is consistent with the findings by Randall et al. (2012) who used three frequencies $(840 \mathrm{MHz}, 1.4 \mathrm{GHz}$ and $2.3 \mathrm{GHz}$ ) for their spectral index calculation and extend their findings to fainter flux limits. Ibar et al. (2009) come to the same conclusion, too.

A flattening of the spectral index with lower flux density levels, as e.g. seen by Zhang et al. (2003), was initially suspected by Windhorst et al. (1993). A major line of argument is that, at sub-mJy levels, a change in population takes place: at mJy levels, the radio sky is completely dominated by AGN (see e.g. Padovani et al. 2011), so the dominant emission mechanism is non-thermal synchrotron radiation. An intrinsically flatter spectrum would e.g. be produced by dominating emission due to thermal bremsstrahlung, but this makes up only a marginal fraction even in starburst galaxies (see Condon 1992). In addition, recent work by several authors indicate that the transition from AGN-dominated to SF-dominated radio sources takes place below a flux density of $100 \mu \mathrm{Jy}$. For instance, Padovani et al. (2011) argue that AGN still dominate the source population down to $100 \mu \mathrm{Jy}$ and Smolčić et al. (2008) claim that the fraction of SF-powered radio sources has a nearly constant value of about $40 \%$ for flux levels between $50 \mu \mathrm{Jy}$ and $700 \mu \mathrm{Jy}$. A similar result is obtained by Seymour et al. (2008) investigating the differential number counts in the 13 Hour XMM-Newton/Chandra Deep Field Survey. Hence, this change in the faint radio population cannot account for our findings since we do not reach such faint flux density levels.

With our catalogue of 631 sources with spectral indices, we now focus on the application of the spectral index in terms of its predictive quality. Since it is a quantity which is relatively easy to measure, the spectral index would be a useful tool to characterise sources in large-scale surveys, if a dependence on other properties of interest such as source type, redshift or other scaling relations can be established.

\subsection{Spectral indices as object type discriminator}

The radio spectral index is commonly regarded as a possible tool for discriminating different radio source types, hence emission mechanisms. For instance, Huynh et al. (2007) select AGN based on the assumption that, at low frequencies, only AGN can produce flat or inverted spectral indices because of selfabsorption in an optically thick medium. Hence they propose a cut between $\alpha=-0.3$ and $\alpha=0$ to classify sources as AGN.

With our source sample, we can examine this classification scheme. We cross-matched all our sources with and without unambiguous $2.3 \mathrm{GHz}$ counterpart to the spectral classifications for ATLAS sources by Mao et al. (2012). This cross-match lead to 132 resultant sources of which we can identify 22 SF galaxies and 98 AGN among our sample with measured spectral indices. The remainder 12 sources could either not be reliably classified by means of their optical spectrum (4 sources), or they only have spectral index upper limits (5 AGN, 3 SF galaxies). We suspect that the reason for most spectroscopically classified sources having actual spectral index measurements is due to the fact that the target sources for spectroscopy were chosen such that stronger radio sources were favoured against weaker ones. Therefore, the spectroscopically classified sample shows a significantly higher mean flux density at $1.4 \mathrm{GHz}$ so that most of these sources are detected at $2.3 \mathrm{GHz}$, too. Hence, since the vast majority of cross-identifications both have reliable spectral classifications and accurately measured spectral indices, we chose to omit the few sources that miss some information and proceed with these 120 sources. A histogram showing the distribution of spectral indices among those 120 sources is presented in Fig. 6.

As one can clearly see, the AGN population shows significantly flatter spectral indices than the SF galaxies with median values of -0.63 for AGN and -0.85 for SF galaxies. Note that there are only two SF galaxy exhibiting a spectral index greater than -0.5 . Therefore, flat or inverted spectral indices can reliably be associated with dominant AGN activity. On the other hand, 


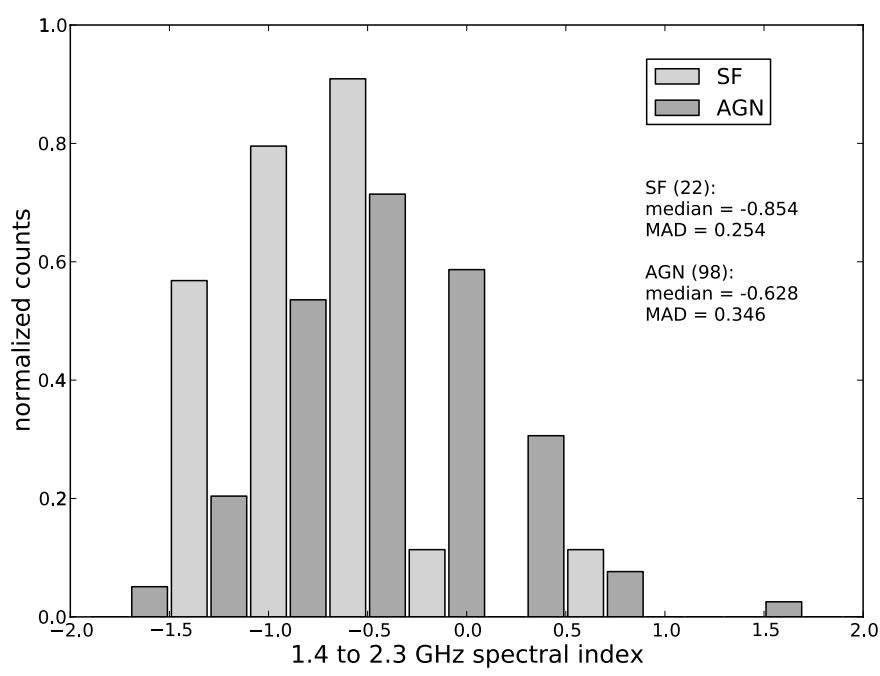

Fig. 6. Histogram illustrating the distribution of spectral indices of spectroscopically classified AGN and SF galaxies.

steep spectral indices (i.e. $\alpha<-1.0$ ) are not associated with any source type as the histogram clearly shows. Therefore, uniquely steep spectra cannot be used as discriminator for source type.

Because the vast majority of (radio) sources detected in contemporary and forthcoming surveys will not have any spectroscopic information, we also searched for correlations between the spectral index and other photometric object type indicators. A popular way of photometrically selecting AGN without being biased by extinction is the use of an infrared colour-colour diagram based on the four channels of the IRAC instrument (Fazio et al. 2004) aboard the Spitzer Space Telescope. Originally proposed by Lacy et al. (2004) and therefore often referred to as the "Lacy diagram", this plot has been further investigated (for an overview see Soifer et al. 2008) so that there are currently reliable template spectral energy distributions (SEDs) available covering the entire optical to MIR regime (e.g. Assef et al. 2010). To construct a "Lacy diagram" for our source sample, we cross-matched the radio sources with the SWIRE catalogue, data release 3, to obtain IRAC flux densities. We found 265 secure identifications where all four IRAC fluxes are available. The corresponding plot is presented in Fig. 7. For comparison, we also plotted all SWIRE-detected sources in the CDF-S and the ELAIS-S1 field as colour-coded, 2-dimensional histogram. The locus of potential AGN in this diagram with respect to various contaminating effects, predominantly line emission by polycyclic aromatic Hydrocarbons (PAHs), was determined by Dasyra et al. (2009) and is indicated in Fig. 7 with a dashed line. We split our source sample in three sub-samples based on their spectral index: steep spectrum sources are classified as having $\alpha<\operatorname{median}(\alpha)-$ MAD, normal spectrum sources satisfy median $(\alpha)-$ MAD $<\alpha<\operatorname{median}(\alpha)+$ MAD and flat/inverted spectrum sources show $\alpha>\operatorname{median}(\alpha)+$ MAD. One can see that flat or inverted spectrum sources are more frequent on the "AGN branch" of the diagram whereas the location of star-forming galaxies (the branch that goes straight up) is more or less avoided by these sources. This "star formation branch" $\left(\log \left(S_{5.8 \mu \mathrm{m}} / S_{3.6 \mu \mathrm{m}}\right)<0\right)$ is dominated by normal and steep spectrum objects with only a very few flat/inverted spectrum sources.

To summarise, the radio spectral index can at least be used to identify a fraction AGN, namely those with optically thick media that cause their radio emission to flatten because of

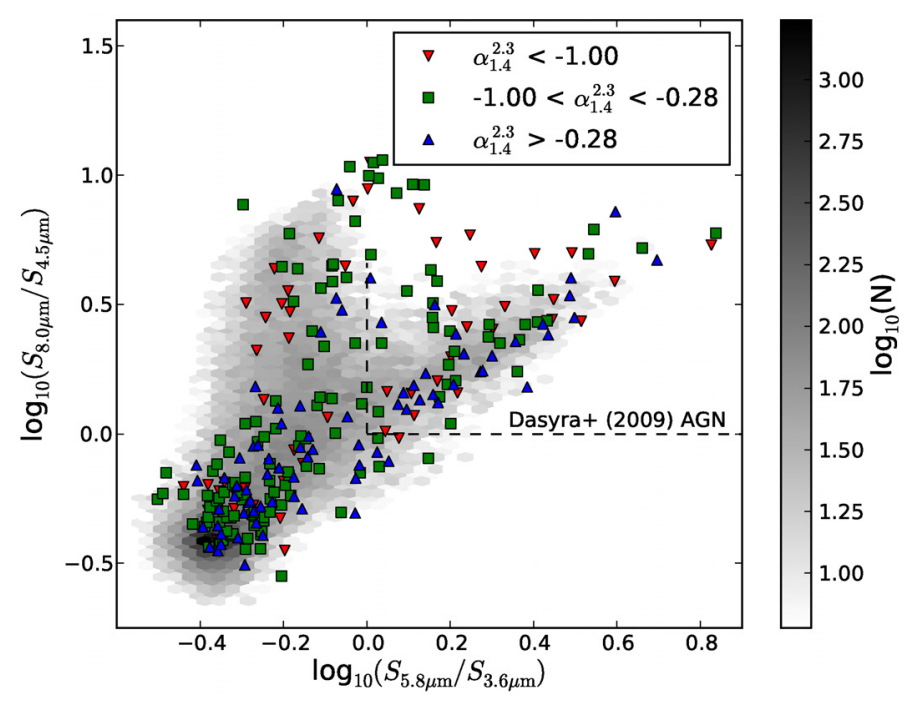

Fig. 7. "Lacy diagram" of our source sample. For comparison, all sources detected in all four IRAC bands within the SWIRE survey of ELAIS-S1 and CDF-S are plotted as 2-dimensional histogram. The black dashed line indicates the locus of AGN in this diagram as proposed by Dasyra et al. (2009).

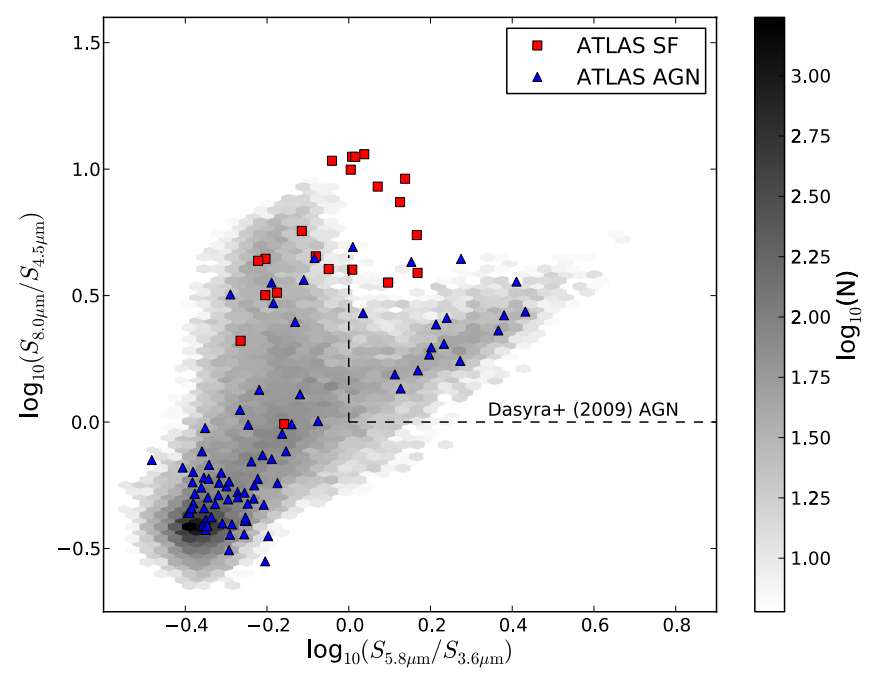

Fig. 8. "Lacy diagram" of our source sample with spectroscopic classification from Mao et al. (2012). As in Fig. 7, all sources detected in all four IRAC bands within the SWIRE survey of ELAIS-S1 and CDF-S are plotted as 2-dimensional histogram. The black dashed line indicates the locus of AGN in this diagram as proposed by Dasyra et al. (2009).

self-absorption energy losses. However, spectral indices are not a strong discriminator for the source type because (i) outliers from this general trend are frequent and (ii) optically thin AGN cannot be separated from SF galaxies with spectral indices. This is further highlighted in Fig. 8 where we plotted the "Lacy diagram" for all ATLAS sources with spectroscopic classification (Mao et al. 2012). It becomes obvious that the "AGN branch" in this plot is really occupied by AGN only whereas the "star formation branch" both hosts SF galaxies and AGN. Hence, also a selection based on MIR colour criteria leads to the result that a AGN can be reliably selected without contamination by star forming objects. The other way round, selecting SF galaxies only without AGN contamination, is not possible by means of MIR colour selection, similar to a selection based on radio spectral indices. 


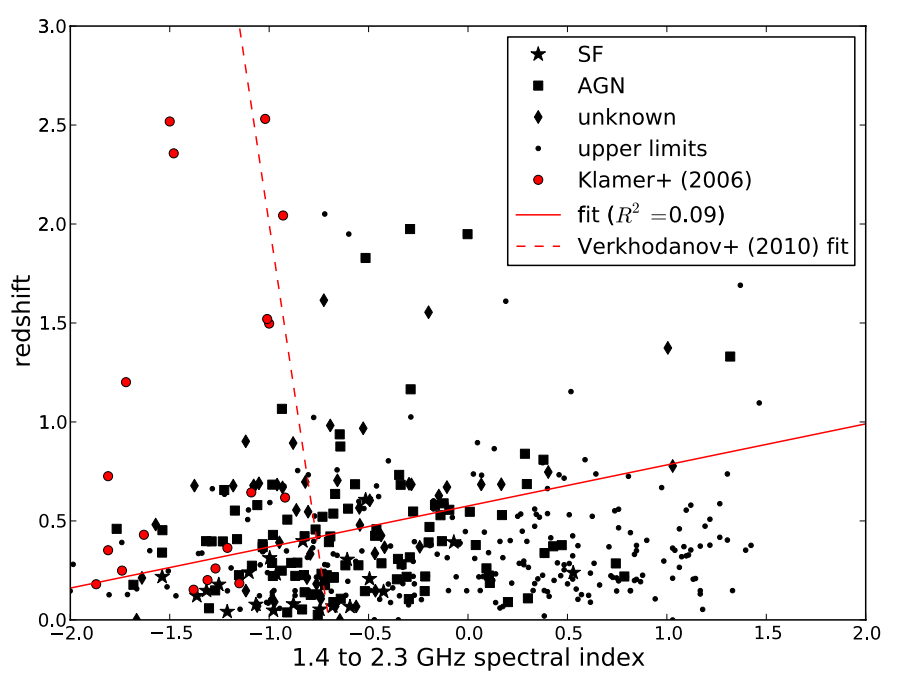

Fig. 9. Redshift versus spectral index plot for all sources with spectroscopic redshift in our sample. Sources with an actual measurement of $\alpha$ are split in spectroscopic classes (AGN: black square, SF: black star, unknown: black diamond). Sources with an upper limit on $\alpha$ only (black dots) were not split up. Note that $R^{2}=0.09$ for the fit to our data points (black symbols) indicates highly uncorrelated quantities. A slight correlation is usually assumed when $R^{2}>0.5$ with good correlation for $R^{2}>0.85$.

\subsection{The $z-\alpha$ relation}

The $z-\alpha$ relation is an empirical relation seen between the spectral index of radio sources in a flux-limited sample and their redshifts. It states that higher-redshift radio sources tend to exhibit steeper spectral indices, typically with $\alpha<-1$. It was found by various authors using different large-scale radio surveys at the Jy and mJy level, see e.g. Athreya \& Kapahi (1998), De Breuck et al. (2000, 2001, 2006), Klamer et al. (2006), Afonso et al. (2011), and Ker et al. (2012). The first quantitative formulation of this relation was given by Verkhodanov \& Khabibullina (2010) using a large sample ( 2500) of distant radio galaxies selected from various surveys across the entire sky. Above a redshift of 1 , they find clear evidence for such a correlation and quantify it by a linear fit $\alpha=(-0.70 \pm 0.02)+(-0.15 \pm 0.01) z$ out to $z \sim 5$. The currently favoured explanation for this effect is based on cosmic expansion (Klamer et al. 2006): at higher redshifts, when the universe was denser on average than at present, the radio jets of high- $z$ AGN had to penetrate into a denser intergalactic medium where they are compressed, similar to the jets of radio galaxies located in the cores of dense clusters in the nearby universe. This compression then leads to the characteristic steepening of the spectral index due to synchrotron and inverse-Compton energy losses of the relativistic electrons.

With the spectroscopic information from Mao et al. (2012), we have investigated the $z-\alpha$ relation in our sub-mJy sample. From our initial 631 sources with measured spectral index, $169(27 \%)$ have spectroscopic redshifts. In addition, we have 236 sources with spectroscopic redshift and an upper limit on $\alpha$ only. A plot of these redshifts versus the spectral index or the upper limits, respectively, is shown in Fig. 9. We again note that the 169 sources with spectroscopic redshifts and actual spectral index measurements are not affected by sensitivity bias (see Sect. 4) since they all have $1.4 \mathrm{GHz}$ flux densities in excess of $0.8 \mathrm{mJy}$ and hence spectral indices of at least -2.0 can be measured for them, given our $2.3 \mathrm{GHz}$ sensitivity.
Surprisingly, in this diagram, there is no obvious correlation between spectral index and redshift. Note that this result is also independent of the spectroscopic classification of the objects. But since radio sources with significant redshift are, due to the sensitivity limits of currently available telescopes, all AGN-powered (even a galaxy forming stars at a very high rate of $1000 M_{\odot} \mathrm{yr}^{-1}$ would be undetectable within ATLAS for any redshift $z>1.2$ ), the $z-\alpha$ relation is yet only investigated for AGN.

This finding (the absence of a $z-\alpha$ relation) contradicts the contemporary literature, and we offer two potential explanations: First, our sample of sources is very small (only 9 sources) for the redshift range $z>1$ were the $z-\alpha$ relation becomes important (see Verkhodanov \& Khabibullina 2010). Hence, our findings rely on small number statistics and have therefore to be treated with care. Second, there could be a change in the radio source population between our sub-mJy sample and the samples used in other work which are mainly much more luminous sources with flux densities of several tens to hundreds of mJy (despite their significant redshifts, so these sources are very luminous). We also point out that Ker et al. (2012) found only a slight correlation between spectral index and redshift. They argue that the relation becomes fully applicable only for resolved sources, if the explanation is correct that the $z-\alpha$ relation arises from high-redshift radio lobes working against the denser intergalactic medium. This reasoning is supported by our findings because nearly all of our sources are compact. In order to effectively select potential high-z radio sources, Ker et al. (2012) suggest to use the relation between $K$-band flux density (or also Spitzer $3.6 \mu \mathrm{m}$ IRAC channel) and redshift which is much tighter than the traditional $z-\alpha$ relation, see also Norris et al. (2011a). This relation was also investigated by Middelberg et al. (2011) to select a certain class of objects which is suspected to be located at very high redshifts $(z>2)$, the so-called Infrared-Faint Radio Sources (IFRS).

According to Zinn et al. (2011), a source is deemed an IFRS if its measured ratio between radio $(20 \mathrm{~cm})$ and nearinfrared (NIR, mostly Spitzer IRAC $3.6 \mu \mathrm{m}$ ) flux density exceeds a cutoff-value of 500 and, at the same time, its NIR flux density is less than $30 \mu \mathrm{Jy}$. Those extreme sources (often exhibiting no $3.6 \mu \mathrm{m}$ counterpart at all, even in ultra-deep or stacked NIR images with noise levels reaching the $100 \mathrm{nJy}$ level) are very difficult to investigate, in particular regarding their redshift, since optical spectroscopy can only be done for such IFRS that at least have moderately bright optical counterparts. Therefore, we only have very few sources in our sample with spectroscopic redshifts that meet at least the first IFRS criterion. A real IFRS meeting both criteria is not in our sample. A plot illustrating the $S_{20 \mathrm{~cm}} / S_{3.6 \mu \mathrm{m}}$ flux ratios of our source sample with respect to redshift is shown in Fig. 10. Contrary to the $z-\alpha$ relation in Fig. 9, there is a clear trend of increasing redshift with increasing $S_{20 \mathrm{~cm}} / S_{3.6 \mu \mathrm{m}}$ flux ratio. Although the scatter in the relation is fairly large, in particular for high flux ratios, this relation seems to be a much better estimator for the redshift of a source than the classical $z-\alpha$ relation. This is in very good agreement with the findings of Ker et al. (2012) who also see only a marginal correlation of the spectral index with redshift but state that by far the most efficient selection criterion for high- $z$ sources in complete samples is based on $K$-band magnitude.

We conclude that our data do not confirm the $z-\alpha$ relation, at least for faint radio sources. This may have several explanations: On the one hand, the popular belief that the $z-\alpha$ relation is caused by the denser intergalactic medium (IGM) at higher redshifts against which the radio jets of powerful sources have 


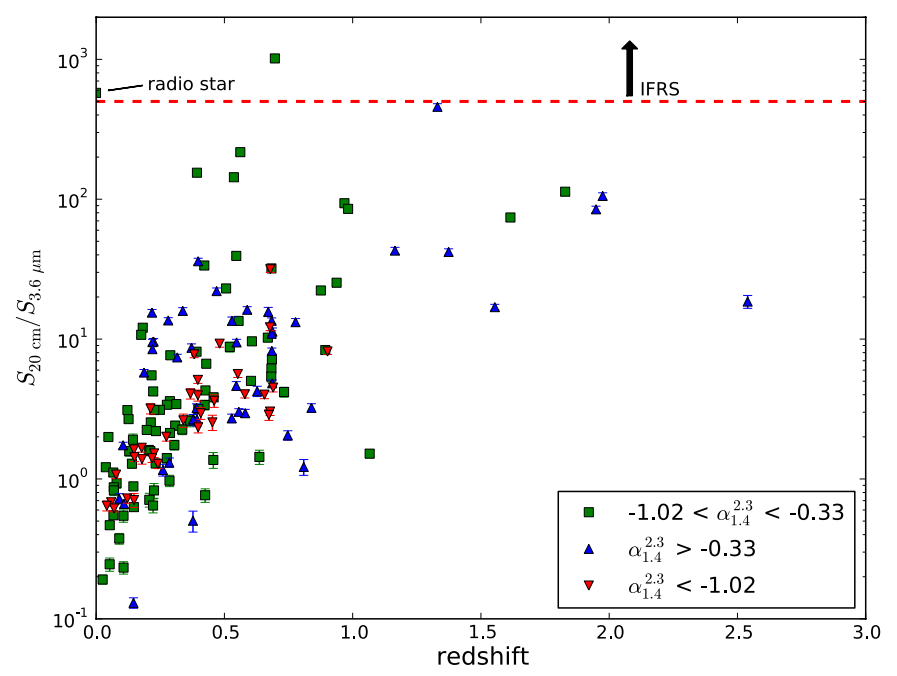

Fig. 10. $S_{20 \mathrm{~cm}} / S_{3.6 \mu \mathrm{m}}$ flux ratios for our spectral index source sample. Note the clear but not very tight trend of increasing redshift with increasing $S_{20 \mathrm{~cm}} / S_{3.6 \mu \mathrm{m}}$. The limiting value for IFRS is shown as red dashed line.

to work and thereby lose energy (Klamer et al. 2006), may not be applicable to faint sources since they are mostly compact. For instance, the simulations used by Raccanelli et al. (2012) predict that compact Fanaroff-Riley type I (FR I) sources are more frequent at any redshift greater than 1 by several orders of magnitude compared to extended FR II sources. Therefore, the overall radio population, at least at the sub-mJy level, should mainly consist of such compact FR I sources for which the Klamer et al. (2006) explanation is less suitable. Furthermore, the $z-\alpha$ relation may only be an apparent correlation. Nearly all radio spectra of the highest- $z(z>4)$ radio sources known to date are significantly curved or even peaked at observed-frame frequencies of $\$ 500 \mathrm{MHz}$ (Ker et al. 2012, their Fig. 5), a behaviour that is indicative for either young sources or sources with recent merger activity (e.g. O'Dea 1998; Randall et al. 2011). Hence, spectral indices calculated between $1.4 \mathrm{GHz}$ and another radio frequency commonly in use today, e.g. $610 \mathrm{MHz}$ or $5 \mathrm{GHz}$, measure the high-frequency flank of such a peaked spectrum which yields increasingly steep spectral indices the more the radio spectrum is curved or peaked. Therefore, sources selected by a steep spectral index are predominantly of these two types (either young or merger). Because those sources are more frequent at higher redshifts when the universe was denser (more merger) and younger (more young sources), it only appears that a selection based on steep spectral indices yields high-redshift objects despite the real astrophysical phenomenon that is selected is completely different, namely young or merger sources.

\subsection{Spectral indices and the radio-IR correlation}

The radio-IR correlation is deemed to be one of the tightest correlations in astrophysics. It states that the radio emission of a galaxy is positively correlated with its mid- and far-infrared emission due to recent star-formation activity. The IR emission in this scenario is produced directly by the star-formation processes whereas the radio emission comes from supernova remnants related to the star-formation event. After its discovery in the late 1980s (e.g. de Jong et al. 1985; Condon et al. 1991), it was subjected to intensive studies while the technology of infrared telescopes advanced (Appleton et al. 2004) and is

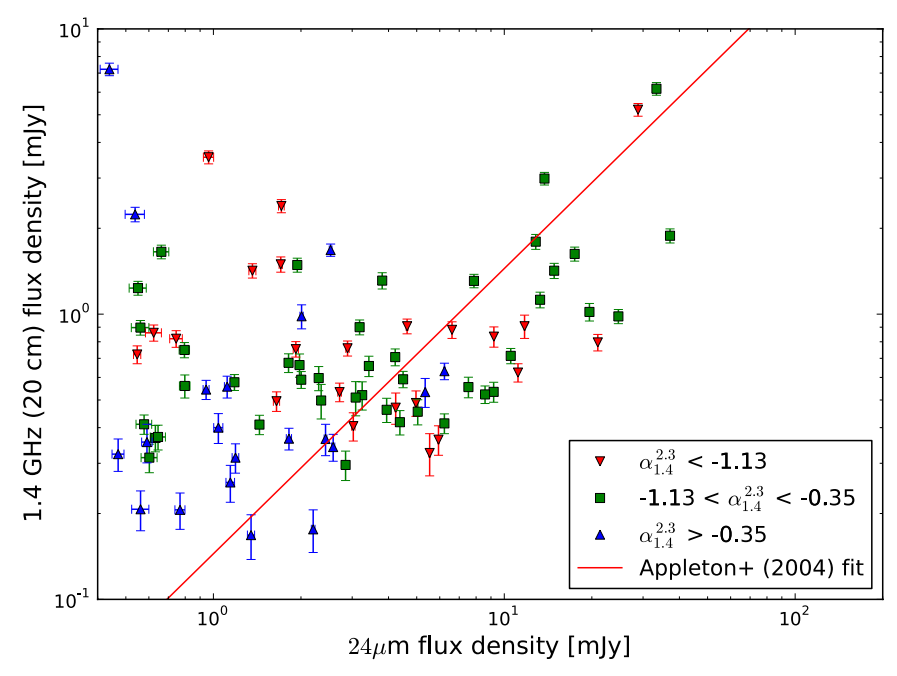

Fig. 11. Diagram illustrating the correlation between the flux densities at $24 \mu \mathrm{m}$ and $20 \mathrm{~cm}(1.4 \mathrm{GHz})$. The sources are divided in three spectral index bins according to $\alpha<\operatorname{median}(\alpha)-\operatorname{MAD}$, median $(\alpha)-\operatorname{MAD}<$ $\alpha<\operatorname{median}(\alpha)+$ MAD and $\alpha>\operatorname{median}(\alpha)+$ MAD where MAD is the median absolute deviation of the spectral index of the entire $24 \mu$ m-detected sample.

nowadays proven to hold over a wide range in both flux density (Boyle et al. 2007; Garn \& Alexander 2009) and redshift (Huynh et al. 2010; Mao et al. 2011).

We here use co-located Spitzer observations at $24 \mu \mathrm{m}$ from the SWIRE survey (Lonsdale et al. 2003) to investigate the dependence of the correlation parameter, $q_{24} \equiv$ $\log \left(S_{24 \mu \mathrm{m}} / S_{20 \mathrm{~cm}}\right)$ on the radio spectral index. Matching our initial sample of 631 radio sources to the SWIRE IR catalogue with a match radius of $5^{\prime \prime}, 145$ counterparts were identified. Because the radio-IR correlation should only hold for star-forming objects, we excluded all known AGN among these sources based on the spectral classifications by Mao et al. (2012) and other AGN indicators like morphology which was done in Norris et al. (2006) and Middelberg et al. (2008). After this rejection procedure, we were left with a total of 86 sources with secure $24 \mu \mathrm{m}$ detection and no signs of AGN activity. A plot showing $S_{24 \mu \mathrm{m}}$ versus $S_{20 \mathrm{~cm}}$ for these objects is presented in Fig. 11 .

The correlation in our source sample is, at least for their slope, well described by the fit of Appleton et al. (2004) which was obtained using SWIRE data but over many fields, not just ELAIS-S1 and CDF-S. The few sources that lie at the top-left corner of the diagram, exhibiting an excess of radio emission, are leftover AGN which were not removed from the sample during the AGN rejection step.

Calculating the correlation parameter $q_{24}$ for our sample, we find a median value of 0.68 with a fairly large spread of $\mathrm{MAD}=0.36$. When plotting the individual values of $q_{24}$ against the spectral indices of the sample (Fig. 12), we see no clear correlation between the two quantities.

Nevertheless, a general trend could be found that sources with a spectral index steeper than the median value show a much larger spread in $q_{24}$ values than sources with a spectral index flatter than the median. This is increasingly surprising with the results of Sect. 4.2 where we found that flat or inverted spectral indices are indicative for AGN-powered sources. Since AGN should not obey the radio-IR correlation, this tightening of the scatter in $q_{24}$ with increasing spectral index is still to be investigated. One possible explanation could arise from the findings 


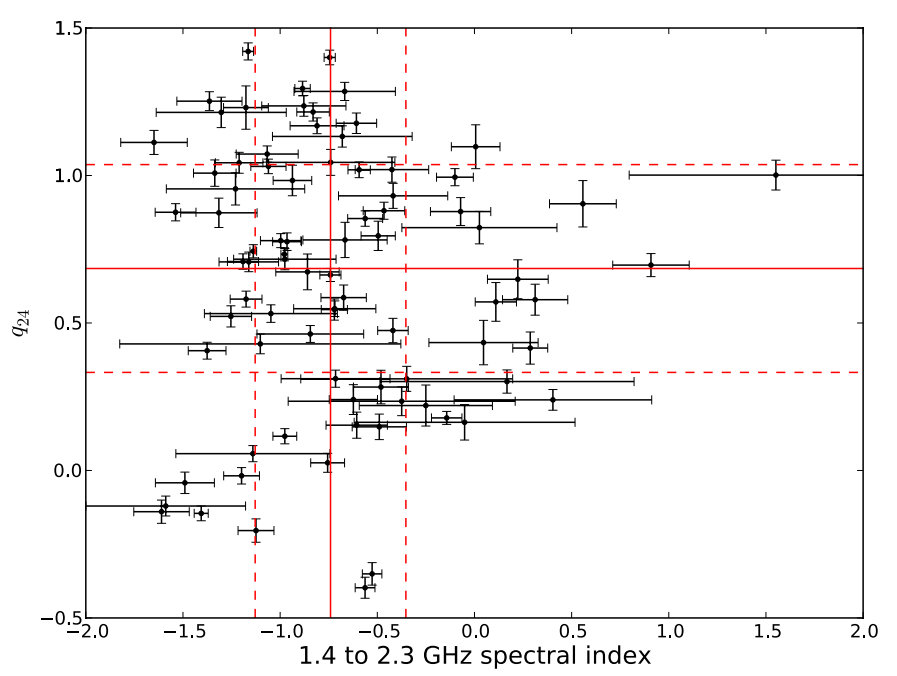

Fig. 12. $q_{24}$ values of our source sample plotted against spectral index. The solid red lines indicate the median of the two quantities while the dashed lines indicate the spread in terms of (median \pm MAD).

of Padovani et al. (2011) who investigated star forming galaxies, radio-loud and radio quiet AGNs in the CDF-S. They found that regarding their luminosity function and its evolution, radioquiet AGN are pretty much similar to star forming galaxies and not to their radio-loud siblings. Although we aimed to exclude AGN from the sample discussed in this section based on indicators such as radio morphology or spectroscopy, we predominantly eliminated radio-loud AGN and several radio-quiet AGN may still contaminating the sample (as suggested by the few flat and inverted spectrum sources in Fig. 12). That these radio-quiet AGN do obey the radio-IR correlation is a natural consequence of the findings of Padovani et al. (2011) and already discussed in their work.

In summary, we conclude that the radio-IR correlation is not affected by the radio spectral indices of sources in a flux-limited sample. This is yet another proof for their remarkable universality which has been shown for redshift, flux density levels and now also for radio spectral properties.

\section{Summary and outlook}

We have conducted deep ( $\sigma=70-80 \mu \mathrm{Jy})$ radio observations of the ELAIS-S1 and CDF-S regions at $2.3 \mathrm{GHz}$ using the ATCA to supplement the sources detected at $1.4 \mathrm{GHz}$ within ATLAS with spectral index information. Our final catalogue with sources well detected at both frequencies and therefore with high quality spectral indices comprise a total of 631 sources and is available at the CDS. The median spectral index of our distribution is -0.73 with a median absolute deviation (MAD) of 0.35 . We have investigated the properties of the population with respect to spectral index and tested correlations between the spectral index and various other quantities. Our main findings are as follows:

1. We do not find evidence for a flattening of the spectral index towards fainter (several $100 \mu \mathrm{Jy}$ ) flux densities. This is in good agreement with the majority of previous work by other authors (e.g. Randall et al. 2012; Ibar et al. 2009). Since we also find that star-forming galaxies tend to have steeper radio spectra compared to AGN, we can support the current notion that star-forming galaxies become the dominant type of radio sources only at flux densities below $100 \mu \mathrm{Jy}$ since our sample does not reach such low flux densities.

2. Considering the spectral index as discriminator for the object type, we find that flat- or inverted-spectrum sources are mostly AGN while SF galaxies show significantly steeper spectra with a median spectral index of -0.85 (AGN median spectral index is -0.68). This flattening is caused by selfabsorption losses within optically thick AGN under physical conditions which are not in general met in SF galaxies. However, there is a significant number of outliers which affects the selection of AGN based on spectral indices. Furthermore, optically thin AGN exhibiting the canonical spectral index of -0.7 for synchrotron radiation can not be separated from SF galaxies. Therefore, we conclude that the spectral index is not a strong discriminator for the source type but can be helpful if there are no other, more sophisticated diagnostics.

3. We investigate the $z-\alpha$ relation which states that highredshift radio galaxies show steeper spectral indices. The relation cannot be confirmed with our data. We suspect that this is explained either by the fact that the currently favoured explanation for the $z-\alpha$ relation (Klamer et al. 2006) is not applicable to the sub-mJy population or because of the complete failure of the $z-\alpha$ relation which may only be an apparent correlation. Since most high- $z$ radio galaxies show curved or even peaked radio spectra, indicative for young or merger sources, a selection based on steep spectral indices may predominantly select those sources. But because both young and merger sources are more frequent at high redshifts when the universe was denser and younger, it appears to select high- $z$ sources.

4. We find no evidence for a dependence of the radio-IR correlation on radio spectral index. This therefore widens the universality of this correlation which is already known to hold over a wide range of flux densities and redshifts. However, we do see a trend for the scatter of $q_{24}$ which is larger for sources with steep spectral indices and becomes smaller while going to positive spectral indices.

In conclusion, we want to point out that radio spectral indices can be useful to characterise a radio-selected sample, but since there are only rough correlations with other source properties, the predictive power of spectral indices may have been overrated in the past. This is unfortunate, in particular for future allsky radio surveys such as the Evolutionary Map of the Universe (EMU, Norris et al. 2011b) to be carried out with the Australia Square Kilometre Array Pathfinder (ASKAP) telescopes in the southern sky or similar surveys in the northern sky planned to be done with the Low Frequency Array (LOFAR, Morganti et al. 2010) or the upgraded Westerbork Synthesis Radio Telescope when equipped with the new focal plane array system Apertif (Rottgering et al. 2011). A significant fraction of the sources detected by these surveys at a typical noise level of $10 \mu \mathrm{Jy}$ will have no other than radio data available, even considering future surveys such as Pan-Starrs (Kaiser et al. 2010), SkyMapper (Keller et al. 2007), and the Large Synoptic Survey Telescope (Ivezic et al. 2008) in the optical or the Wide-field Infrared Survey Explorer (WISE, Wright et al. 2010) and ESO public surveys with the VISTA and VST telescopes (Arnaboldi et al. 2007) in the infrared.

Acknowledgements. We thank our anonymous referee for many insightful comments and suggestions which improved the scientific validity of this paper a lot. P.C.Z. acknowledges funding by the Ruhr-University Research School. 


\section{References}

Afonso, J., Bizzocchi, L., Ibar, E., et al. 2011, ApJ, 743, 122 Appleton, P. N., Fadda, D. T., Marleau, F. R., et al. 2004, ApJS, 154, 147 Arnaboldi, M., Neeser, M. J., Parker, L. C., et al. 2007, The Messenger, 127, 28 Assef, R. J., Kochanek, C. S., Brodwin, M., et al. 2010, ApJ, 713, 970 Athreya, R. M., \& Kapahi, V. K. 1998, J. Astrophys. Astron., 19, 63 Balestra, I., Mainieri, V., Popesso, P., et al. 2010, A\&A, 512, A12 Bondi, M., Ciliegi, P., Venturi, T., et al. 2007, A\&A, 463, 519 Boyle, B. J., Cornwell, T. J., Middelberg, E., et al. 2007, MNRAS, 376, 1182 Condon, J. J. 1992, ARA\&A, 30, 575

Condon, J. J. 1997, PASP, 109, 166

Condon, J. J., Anderson, M. L., \& Helou, G. 1991, ApJ, 376, 95

Condon, J. J., Cotton, W. D., Greisen, E. W., et al. 1998, AJ, 115, 1693

Dasyra, K. M., Yan, L., Helou, G., et al. 2009, ApJ, 701, 1123

De Breuck, C., van Breugel, W., Röttgering, H. J. A., \& Miley, G. 2000, A\&AS, 143,303

De Breuck, C., van Breugel, W., Röttgering, H., et al. 2001, AJ, 121, 1241

De Breuck, C., Klamer, I., Johnston, H., et al. 2006, MNRAS, 366, 58

de Jong, T., Klein, U., Wielebinski, R., \& Wunderlich, E. 1985, A\&A, 147, L6

Fazio, G. G., Hora, J. L., Allen, L. E., et al. 2004, ApJS, 154, 10

Feigelson, E. D., \& Nelson, P. I. 1985, ApJ, 293, 192

Garn, T., \& Alexander, P. 2009, MNRAS, 394, 105

Hopkins, A. M., Afonso, J., Chan, B., et al. 2003, AJ, 125, 465

Huynh, M. T., Jackson, C. A., \& Norris, R. P. 2007, AJ, 133, 1331

Huynh, M. T., Gawiser, E., Marchesini, D., Brammer, G., \& Guaita, L. 2010, ApJ, 723, 1110

Ibar, E., Ivison, R. J., Biggs, A. D., et al. 2009, MNRAS, 397, 281

Ivezic, Z., Axelrod, T., Brandt, W. N., et al. 2008, Serbian Astron. J., 176, 1 Jaeger, S. 2008, in Astronomical Data Analysis Software and Systems XVII, eds. R. W. Argyle, P. S. Bunclark, \& J. R. Lewis, ASP Conf. Ser., 394, 623 Kaiser, N., Burgett, W., Chambers, K., et al. 2010, in SPIE Conf. Ser., 7733 Keller, S. C., Schmidt, B. P., Bessell, M. S., et al. 2007, PASA, 24, 1

Ker, L. M., Best, P. N., Rigby, E. E., Röttgering, H. J. A., \& Gendre, M. A. 2012, MNRAS, 2178

Klamer, I. J., Ekers, R. D., Bryant, J. J., et al. 2006, MNRAS, 371, 852

Komatsu, E., Smith, K. M., Dunkley, J., et al. 2011, ApJS, 192, 18 Lacy, M., Storrie-Lombardi, L. J., Sajina, A., et al. 2004, ApJS, 154, 166 Lonsdale, C. J., Smith, H. E., Rowan-Robinson, M., et al. 2003, PASP, 115, 897 Mao, M. Y., Huynh, M. T., Norris, R. P., et al. 2011, ApJ, 731, 79
Mao, M. Y., Norris, R. P., Sharp, R. G., \& Middelberg, E. 2012, MNRAS, accepted

Middelberg, E. 2006, PASA, 23, 64

Middelberg, E., Norris, R. P., Cornwell, T. J., et al. 2008, AJ, 135, 1276

Middelberg, E., Norris, R. P., Hales, C. A., et al. 2011, A\&A, 526, A8

Morganti, R., Rottgering, H., Snellen, I., et al. 2010 [arXiv: 1001.2384]

Moster, B. P., Somerville, R. S., Newman, J. A., \& Rix, H.-W. 2011, ApJ, 731, 113

Norris, R. P., Afonso, J., Appleton, P. N., et al. 2006, AJ, 132, 2409

Norris, R. P., Afonso, J., Cava, A., et al. 2011a, ApJ, 736, 55

Norris, R. P., Hopkins, A. M., Afonso, J., et al. 2011b, PASA, 28, 215

O'Dea, C. P. 1998, PASP, 110, 493

Owen, F. N., \& Morrison, G. E. 2008, AJ, 136, 1889

Padovani, P., Miller, N., Kellermann, K. I., et al. 2011, ApJ, 740, 20

Popesso, P., Dickinson, M., Nonino, M., et al. 2009, A\&A, 494, 443

Prandoni, I., Parma, P., Wieringa, M. H., et al. 2006, A\&A, 457, 517

Raccanelli, A., Zhao, G.-B., Bacon, D. J., et al. 2012, MNRAS, 424, 801

Randall, K. E., Hopkins, A. M., Norris, R. P., \& Edwards, P. G. 2011, MNRAS, 416, 1135

Randall, K., Hopkins, A., Norris, R., et al. 2012, MNRAS, 421, 1644

Rix, H.-W., Barden, M., Beckwith, S. V. W., et al. 2004, ApJS, 152, 163

Rottgering, H., Afonso, J., Barthel, P., et al. 2011, J. Astrophys. Astron., 32, 557

Sacchi, N., La Franca, F., Feruglio, C., et al. 2009, ApJ, 703, 1778

Sault, R. J., Teuben, P. J., \& Wright, M. C. H. 1995, in Astronomical Data Analysis Software and Systems IV, eds. R. A. Shaw, H. E. Payne, \& J. J. E. Hayes, ASP Conf. Ser., 77, 433

Schinnerer, E., Sargent, M. T., Bondi, M., et al. 2010, ApJS, 188, 384

Seymour, N., Dwelly, T., Moss, D., et al. 2008, MNRAS, 386, 1695

Smolčić, V., Schinnerer, E., Scodeggio, M., et al. 2008, ApJS, 177, 14

Soifer, B. T., Helou, G., \& Werner, M. 2008, ARA\&A, 46, 201

Verkhodanov, O. V., \& Khabibullina, M. L. 2010, Astron. Lett., 36, 7

Windhorst, R. A., van Heerde, G. M., \& Katgert, P. 1984, A\&AS, 58, 1

Windhorst, R. A., Fomalont, E. B., Partridge, R. B., \& Lowenthal, J. D. 1993, ApJ, 405, 498

Wolf, C., Meisenheimer, K., Rix, H.-W., et al. 2003, A\&A, 401, 73

Wright, E. L., Eisenhardt, P. R. M., Mainzer, A. K., et al. 2010, AJ, 140, 1868

Zhang, X.-Z., Reich, W., Reich, P., \& Wielebinski, R. 2003, Chin. J. Astron. Astrophys., 3, 347

Zinn, P.-C., Middelberg, E., \& Ibar, E. 2011, A\&A, 531, A14 